\title{
MINIREVIEW
}

\section{Endothelial adherens junctions and the actin cytoskeleton: an 'infinity net'?}

\author{
Maria Grazia Lampugnani* \\ See research article http://www.biomedcentral.com/1741-7007/8/11
}

\begin{abstract}
A recent paper in BMC Biology reports that actin stress fibers in adjacent cultured endothelial cells are linked through adherens junctions. This organization might provide a super-cellular network that could enable coordinated signaling and structural responses in endothelia.
\end{abstract}

The title of this minireview is inspired by the work of the Japanese artist Yayoi Kusama, who paints wonderful networks as one of her favorite subjects. As an artist, she can evoke images and concepts that are sometimes beyond her own knowledge: in this case her idea of a continuous network well suits a model of intercellular cytoskeletal linking suggested by a recent paper published in BMC Biology by Millan et al. [1]. Working with cultured human umbilical cord vein endothelial cells they find that actin-based stress fibers in adjacent endothelial cells in confluent culture can become linked through adherens junctions. This organization could, in theory, enable a communication network extending throughout the endothelium, as well as maintaining structural coherence and increasing resistance to stress. This capacity could be crucial to the function and adaptability of the endothelium, which is continuously exposed to changes in blood flow, whether in normal physiologic situations, or during inflammation or angiogenesis.

This is a novel extension of a concept first used for epithelial sheets [2] to endothelial monolayers.

\section{The adherens junction}

The intercellular junctions between endothelial cells maintain endothelial integrity, controlling the movement of solutes between bloodstream and tissues, and the flow

* Correspondence: mariagrazia.lampugnani@ifom-ieo-campus.it IFOM, FIRC Institute of Molecular Oncology, via Adamello, 16-20139 Milan, Italy and Mario Negri Institute of Pharmacology, via La Masa, 19-20156 Milan, Italy of white blood cells between blood and tissues, especially at sites of infection and inflammation. Adherens junctions both mediate adhesion between cells and support the local concentration of scaffolding and signaling molecules. This enables molecular interactions that otherwise would not take place and the segregation of signaling molecules for specific regulation (for a general description of the organization of cell-cell contacts in endothelial cells see [3]). Adherens junctions are based on the transmembrane adhesive receptor that belongs to the cadherin family and a notable and specific feature of the endothelial junctions is the presence of the tissue-specific vascular endothelial (VE)-cadherin. VE-cadherin is constitutively linked through its cytoplasmic tail directly to $\beta$ - or $\gamma$-catenin (plakoglobin) or the catenin p120, and indirectly to $\alpha$-catenin via $\beta$ - or $\gamma$-catenin.

From studies in epithelial and endothelial cells, the conventional view of adherens junctions is that they link through the catenin complex to cortical actin filaments that lie parallel to the cell surface. In their confluent endothelial cell cultures, however, Millan et al. [1] found an additional type of adherens junction organization. They found that staining for junctional components such as VE-cadherin revealed lines of staining running in from the cell surface rather than lying parallel to the cell surface as in conventional adherens junction complexes. The authors call these novel junctions 'discontinuous adherens junctions' to distinguish them from those more familiar from epithelial cells. Further staining for VEcadherin and actin revealed that actin stress fibers running perpendicular to the cell surface were associated with these discontinuous adherens junctions (Figure 1).

\section{Local regulation of actin by adherens junctions in endothelial cells}

At early phases of junction formation, actin filaments organize perpendicularly to the membrane through the local accumulation of actin-nucleating complexes [4]. Further stages of junction maturation are accompanied by the development of actin fibers parallel to the membrane to form a peripheral actin rim ([5] and references therein). It is not clear at present whether the perpendicular actin 


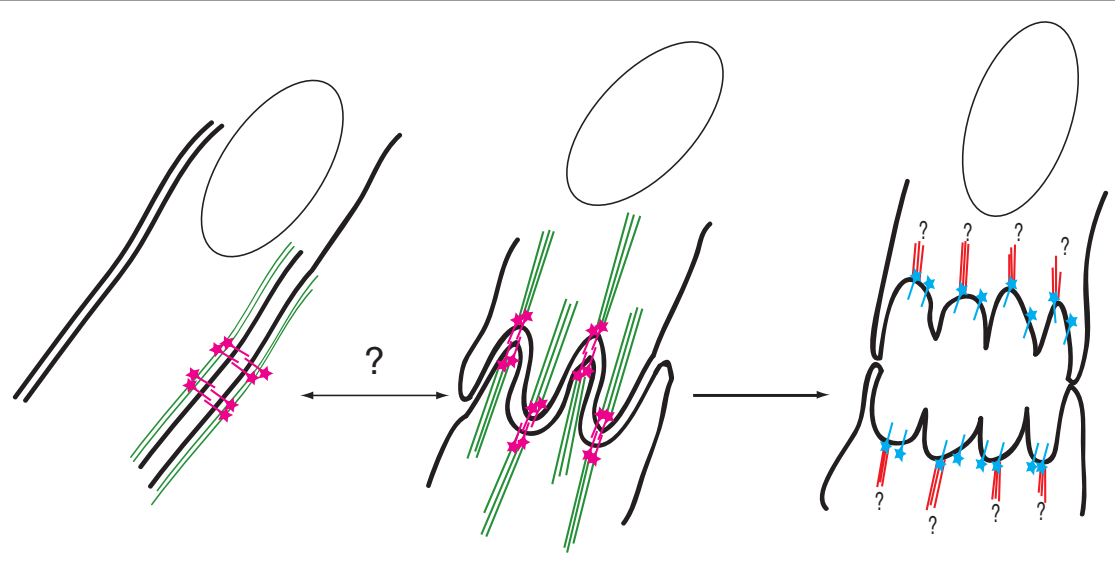

$\begin{gathered}\text { 'Stable' stress fiber } \\ \text { domain }\end{gathered}$

Actin polymerization
Rac activated
Rho activated/signal to Dia

Quiescent endothelium
Contractile response and intercellular gaps

Acto myosin contraction

Rho activated/signal to ROCK

Inflammation,

leukocyte transmigration, angiogenesis

Figure 1. Structural and functional actin filament organization at cell-cell junctions in endothelial cells. In quiescent cells, junctional actin filaments can be oriented either parallel (left-hand image) or perpendicular (center image) to the cell surface. A question mark indicates that it is not known whether these two types of arrangement can interchange, or whether the perpendicular arrangement must arise de novo. Actomyosin stress fibers are anchored to the membrane through adherens junctions [1]. VE-cadherin is required, but the stress fibers are not directly linked to the cadherin-catenin complex. They are possibly tethered to adherens junctions by afadin or other, still unidentified molecules (see Figure 2, and text for details). Subdomains with perpendicular actin fibers, called 'discontinuous adherens junctions' in [1], are dynamic, and undergo constant reorganization. In these areas, actin fibers of adjoining cells are connected through adherens junctions into a supercellular network [1]. In quiescent endothelial cells, actin stress fibers are polymerized through the action of Rac and Rho, Rho signaling through the actin nucleator Dia (diaphanousrelated formin) [7]. Various stimuli activate the endothelium and induce actomyosin contraction at the dynamic domains (right-hand image), with Rho signaling through the protein kinase ROCK [7], and resulting in the opening of intercellular gaps, making the endothelium permeable to fluid and molecules, and facilitating the transmigration of leukocytes. In activated conditions, adherens junctions are also functionally and structurally modified (indicated by the change of the junctional symbol to blue). The molecular details of such alterations are still incompletely understood. The association of actin with adherens junctions is also likely to be modified. It remains to be defined at which molecules/structures (indicated by question marks) anchor the actin fibers at the ends that are not interacting with the adherens junction. This anchoring is critical to allow productive contraction.

fibers described by Millan et al. [1] are residual early fibers or derive by de novo organization. Actin reorganization at cell-cell junctions proceeds through the action of several regulators that concentrate locally (Figure 2).

A crucial molecule in the organization of actin at the adherens junctions is $\alpha$-catenin. The molecular features of the relationship between $\alpha$-catenin and actin have been profoundly revised in recent years (reviewed in [6]). Instead of a 'simple' bridge between junctional cadherin and the actin microfilament cytoskeleton, $\alpha$-catenin is now considered to act as a regulator of actin polymerization. For allosteric reasons $\alpha$-catenin cannot bind at the same time to $\beta$-catenin and actin filaments. Dimeric $\alpha-$ catenin binds with high affinity to actin filaments, and inhibiting Arp $2 / 3$ activity suppresses actin branching. In addition, dimeric $\alpha$-catenin promotes the formation of linear actin cables by activating formin (Figure 2; [6] and references therein).

If $\alpha$-catenin is not the tether between VE-cadherin and actin, which junctional components might be? This is not known at present. Afadin, a cytoplasmic molecule localized at adherens junctions bound to the nectin adhesive receptors, can anchor actin to the plasma membrane and could contribute to the stabilization of the peripheral actin rim $[5,6]$. Which other junctional components might tether actin filaments to adherens junctions remains to be determined.

In addition, $\beta$-catenin has an important role in regulating the behavior of actin. Small GTPases of the Rho family that have crucial effects on the control of 


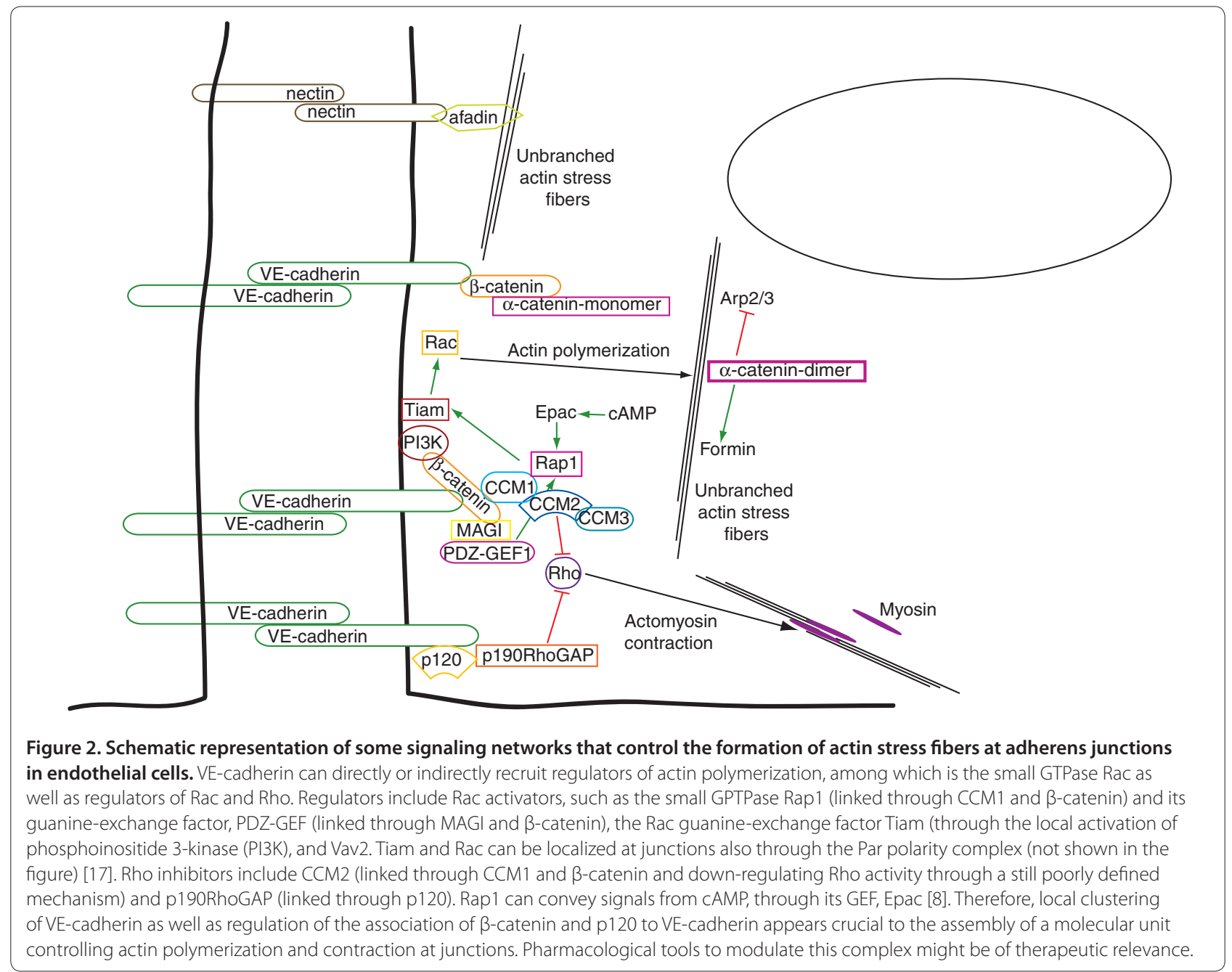

actin polymerization [4] and on the contractility of actomyosin are recruited and regulated at adherens junctions through $\beta$-catenin (Figure 2; see [7] for a comprehensive review of the roles of these GTPases in epithelial cells).

Remodeling or de novo polymerization of actin filaments can be controlled by the GTPases Rac and Rho, while Rho also affects the contractility of actomyosin, the complex of actin and myosin that makes up the contractile stress fibers. Rac signaling at adherens junctions is likely to be downstream of the small GTPase Rap1 [8], which in endothelial cells can be localized at adherens junctions in a complex with VE-cadherin through CCM1 and $\beta$-catenin [9]. In addition, Rap1 can be activated at junctions by PDZ-GEF, recruited locally by the protein MAGI, which is linked to VE-cadherin through $\beta$-catenin [10]. Rap1 can also convey signals from cAMP, through its guanine nucleotide exchange factor (GEF) Epac, to stabilize the barrier properties of the endothelium both in vitro and in vivo [8].
Exaggerated actomyosin contractility has been proposed to be detrimental to the correct function of the endothelium as a barrier to the passage of cells and molecules. This idea is supported by the fact that to maintain junctional stability in the endothelium, the activity of Rho has to be restricted. The specific Rho inhibitor p190Rho-GAP is required in endothelial cells to maintain endothelial integrity and endothelial barrier function. In this case p120 catenin acts as scaffold [11]. Cerebral cavernous malformation protein 2 (CCM2) also inhibits Rho activity [12]. CCM2 is one component of the CCM complex that comprises also CCM1 and CCM3 (Figure 2). Mutation of any of these three molecules can cause the human pathological condition cerebral cavernous malformation. These vascular abnormalities of the brain are fragile and leaky and can cause hemorrhagic stroke and neurological symptoms [13]. Drugs that inhibit Rho, such as simvastatin, have proved effective in correcting increased vascular permeability in mice with a heterozygous mutation of CCM2 [12]. 
It remains to be defined how all these molecular actors can be regulated locally to create the different domains of junctional actin reported by Millan et al. [1].

Finally, to set the observations reported by Millan et al. [1] in a physiological context, an appreciation of the correspondence between in vitro models of endothelial cells and the endothelium in vivo is important. Adherens junctions and stress fibers are readily observed in endothelial cells in vitro. Adherens junctions are present in the endothelium of all types of blood vessels in vivo as observed by in situ immunofluorescence and immunoelectron microscopy for junction-specific molecules [14]. Reports on the organization of actin in the endothelium in vivo are, however, very limited and somewhat controversial $[15,16]$. While suggesting that actin stress fibers can be observed in vivo in endothelial cells, the available data indicate that great care is needed in interpreting the arrangement of actin and its molecular regulation by adherens junctions in vivo both in resting and activated endothelium.

Nevertheless, endothelial adherens junctions seem to be equipped with the molecular components to regulate actin polymerization and actomyosin contraction. The results of Millan et al. [1] suggest that local tuning of the actin cytoskeleton at adherens junction subdomains could allow endothelial cells in the monolayer to behave as a functional unit, withstanding the stress of continuous changes in blood flow that take place in the organism and also locally restricting the stress of junctional and actin rearrangements that occur during inflammation, leukocyte transmigration and angiogenic responses.

Published: 8 April 2010

\section{References}

1. Millan J, Cain RJ, Reglero-Real N, Bigarella C, Marcos-Ramiro B, FernandezMartin L, Correas I, Ridley AJ: Adherens junctions connect stress fibers between adjacent endothelial cells. BMC Biol 2010, 8:11.

2. Cai Y, Sheetz MP: Force propagation across cells: mechanical coherence of dynamic cytoskeletons. Curr Opin Cell Biol 2009, 21:47-50.
3. Dejana E, Orsenigo F, Lampugnani MG: The role of adherens junctions and VE-cadherin in the control of vascular permeability. J Cell Sci 2008, 121:2115-2122.

4. Ridley AJ: Rho GTPases and actin dynamics in membrane protrusions and vesicle trafficking. Trends Cell Biol 2006, 16:522-529.

5. Niessen $C M$, Gottardi $\mathrm{CJ}$ : Molecular components of the adherens junction. Biochim Biophys Acta 2008, 1778:562-571.

6. Pokutta S, Drees F, Yamada S, Nelson WJ, Weis WI: Biochemical and structural analysis of alpha-catenin in cell-cell contacts. Biochem Soc Trans 2008, 36:141-147.

7. Samarin $S$, Nusrat A: Regulation of epithelial apical junctional complex by Rho family GTPases. Front Biosci 2009, 14:1 129-1142.

8. Pannekoek WJ, Kooistra MR, Zwartkruis FJ, Bos UL: Cell-cell junction formation: the role of Rap1 and Rap1 guanine nucleotide exchange factors. Biochim Biophys Acta 2009, 1788:790-796.

9. Glading A, Han J, Stockton RA, Ginsberg MH: KRIT-1/CCM1 is a Rap1 effector that regulates endothelial cell cell junctions. J Cell Bio/ 2007, 179:247-254.

10. Sakurai A, Fukuhara S, Yamagishi A, Sako K, Kamioka Y, Masuda M, Nakaoka Y, Mochizuki N: MAGI-1 is required for Rap1 activation upon cell-cell contact and for enhancement of vascular endothelial cadherin-mediated cell adhesion. Mol Biol Cell 2006, 17:966-976.

11. Wildenberg GA, Dohn MR, Carnahan RH, Davis MA, Lobdell NA, Settleman J, Reynolds AB: p120-catenin and p190RhoGAP regulate cell-cell adhesion by coordinating antagonism between Rac and Rho. Cell 2006 , 127:1027-1039.

12. Whitehead KJ, Chan AC, Navankasattusas S, Koh W, London NR, Ling J, Mayo AH, Drakos SG, Jones CA, Zhu W, Marchuk DA, Davis GE, Li DY: The cerebral cavernous malformation signaling pathway promotes vascular integrity via Rho GTPases. Nat Med 2009, 15:177-184.

13. Labauge P, Denier C, Bergametti F, Tournier-Lasserve E: Genetics of cavernous angiomas. Lancet Neurol 2007, 6:237-244.

14. Baluk P, Fuxe J, Hashizume H, Romano T, Lashnits E, Butz S, Vestweber D, Corada M, Molendini C, Dejana E, McDonald DM: Functionally specialized junctions between endothelial cells of lymphatic vessels. J Exp Med 2007, 204:2349-2362.

15. Nehls V, Drenckhahn D: Demonstration of actin filament stress fibers in microvascular endothelial cells in situ. Microvasc Res 1991, 42:103-112.

16. Thurston G, Baldwin AL, Wilson LM: Changes in endothelial actin cytoskeleton at leakage sites in the rat mesenteric microvasculature. Am J Physiol 1995, 268:H316-329.

17. Lampugnani, MG, Orsenigo F, Rudini N, Maddaluno L, Boulday G, Chapon F, Dejana E: CCM1 regulates vasacular-lumen organization by inducing endothelial polarity. J Cell Sci 2010, in press.

doi:10.1186/jbiol232

Cite this article as: Lampugnani MG: Endothelial adherens junctions and

the actin cytoskeleton: an'infinity net'? Journal of Biology 2010, 9:16. 